\title{
Hypoxia, hypoxia-inducible transcription factors and oxygen-sensing prolyl hydroxylases in bone development and homeostasis
}

\author{
Steve Stegen ${ }^{\mathrm{a}, \mathrm{b}}$ and Geert Carmeliet ${ }^{\mathrm{a}, \mathrm{b}}$
}

\begin{abstract}
Purpose of review
To summarize the role of hypoxia signaling in skeletal cells.

Recent findings

Hypoxia occurs at several stages during bone development. Skeletal cells, like chondrocytes and osteoblasts, respond to this challenge by stabilizing the hypoxia inducible transcription factor HIF, which induces the expression of angiogenic factors and promotes glycolysis. The increased delivery of oxygen and nutrients, together with metabolic adaptations, prevent chondrocyte cell death in the growth plate and promote bone formation by osteoblasts. However, excessive HIF levels have to be avoided during bone development as the resulting metabolic maladaptations cause skeletal dysplasia. Recent studies show that HIF also targets other genes to increase bone mass: it decreases osteoclastogenesis by increasing osteoprotegerin expression and represses sclerostin expression by epigenetic mechanisms, resulting in increased bone formation and decreased resorption. Moreover, increased HIF signaling in osteolineage cells promotes primary and metastatic breast tumor growth, and induces erythropoietin (EPO) production, resulting in polycythemia. Finally, HIF can directly or indirectly through increasing EPO levels, induce the expression and processing of FGF23 and may thereby affect mineral homeostasis and vitamin D metabolism.
\end{abstract}

\section{Summary}

HIF signaling in skeletal cells not only affects their behavior but also influences erythropoiesis and possibly mineral homeostasis.

\section{Keywords}

angiogenesis, erythropoietin, FGF23, hypoxia-inducible factor prolyl hydroxylase, hypoxia, metabolism

\section{INTRODUCTION}

Throughout life, bone is continuously remodeled by the action of bone-forming osteoblasts and boneresorbing osteoclasts to maintain its structural and functional integrity $[1,2]$. Skeletal cell function is regulated not only by hormones, growth factors, signals from the nervous system and mechanical stimulation, but also by the local microenvironment. Like for most other organs and tissues, it is well established that oxygen and nutrients are essential for proper bone cell function. Although the skeleton is a highly vascularized organ [3], particular regions of the bone microenvironment are characterized by surprisingly low oxygen tensions (i.e. hypoxia) $[4,5]$, possibly because of a low blood flow caused by the serial organization of the two capillary systems [6], and because of the high oxygen consumption by hematopoietic marrow cells [5].
Because of the vital importance of oxygen, skeletal cells have developed mechanisms to sense and respond to local oxygen fluctuations, based on hypoxia-inducible transcription factors (HIFs) that execute the cellular response to hypoxia and the HIF prolyl hydroxylase (PHD) oxygen sensors that regulate HIF levels in an oxygen-dependent manner.

aLaboratory of Clinical and Experimental Endocrinology, Department of Chronic Diseases, Metabolism and Ageing and ${ }^{\mathrm{b}}$ Prometheus, Division of Skeletal Tissue Engineering, KU Leuven, Leuven, Belgium

Correspondence to Geert Carmeliet, MD, PhD, Laboratory of Clinical and Experimental Endocrinology, Department of Chronic Diseases, Metabolism and Ageing, KU Leuven, O\&N1 Herestraat 49 box 902, 3000 Leuven, Belgium. Tel: +32 16330 731; fax: +32 16330 718; e-mail: geert.carmeliet@kuleuven.be

Curr Opin Nephrol Hypertens 2019, 28:000-000

DOI:10.1097/MNH.0000000000000508 


\section{KEY POINTS}

- Skeletal cells express components of the hypoxia signaling pathway.

- During endochondral ossification, HIF-1 $\alpha$ controls chondrocyte function but its levels are regulated by the PHD2 oxygen sensor to avoid skeletal dysplasia.

- In postnatal bone, the interaction between PHDs and HIFs controls bone mass and erythropoiesis.

- HIF signaling induces EPO production, which may in turn increase FGF23 secretion and cleavage in bone marrow cells.

The transcription factors HIF-1, HIF-2 and HIF-3, the central mediators of the response to hypoxia, are heterodimers composed of an oxygen-sensitive HIF$\alpha$ subunit and a constitutively expressed HIF- $\beta$ subunit (Fig. 1) [7-9]. In well oxygenated tissues, the half-life of HIF- $\alpha$ is less than $5 \mathrm{~min}$, and this rapid normoxic turnover is facilitated by PHD-mediated hydroxylation of specific residues within a central oxygen-dependent degradation domain. At least three PHD isoforms have been found to hydroxylate HIF- $\alpha$ (termed PHD1, PHD2 and PHD3; encoded in humans by, respectively, EGLN2, EGLN1 and EGLN3) and they are part of a large family of $\alpha$-ketoglutarate $(\alpha \mathrm{KG})$-dependent dioxygenases that require oxygen, iron and $\alpha \mathrm{KG}$ to function. Hydroxylation prompts polyubiquitylation of HIF- $\alpha$ and is essential for the interaction with the von Hippel-Lindau tumor-suppressor protein (pVHL), which is the recognition component of an E3 ubiquitin ligase complex that targets HIF- $\alpha$ for proteolytic degradation. As oxygen levels decrease, PHD-dependent hydroxylation is reduced and HIF- $\alpha$ is stabilized [7-9]. At that time, HIF- $\alpha$

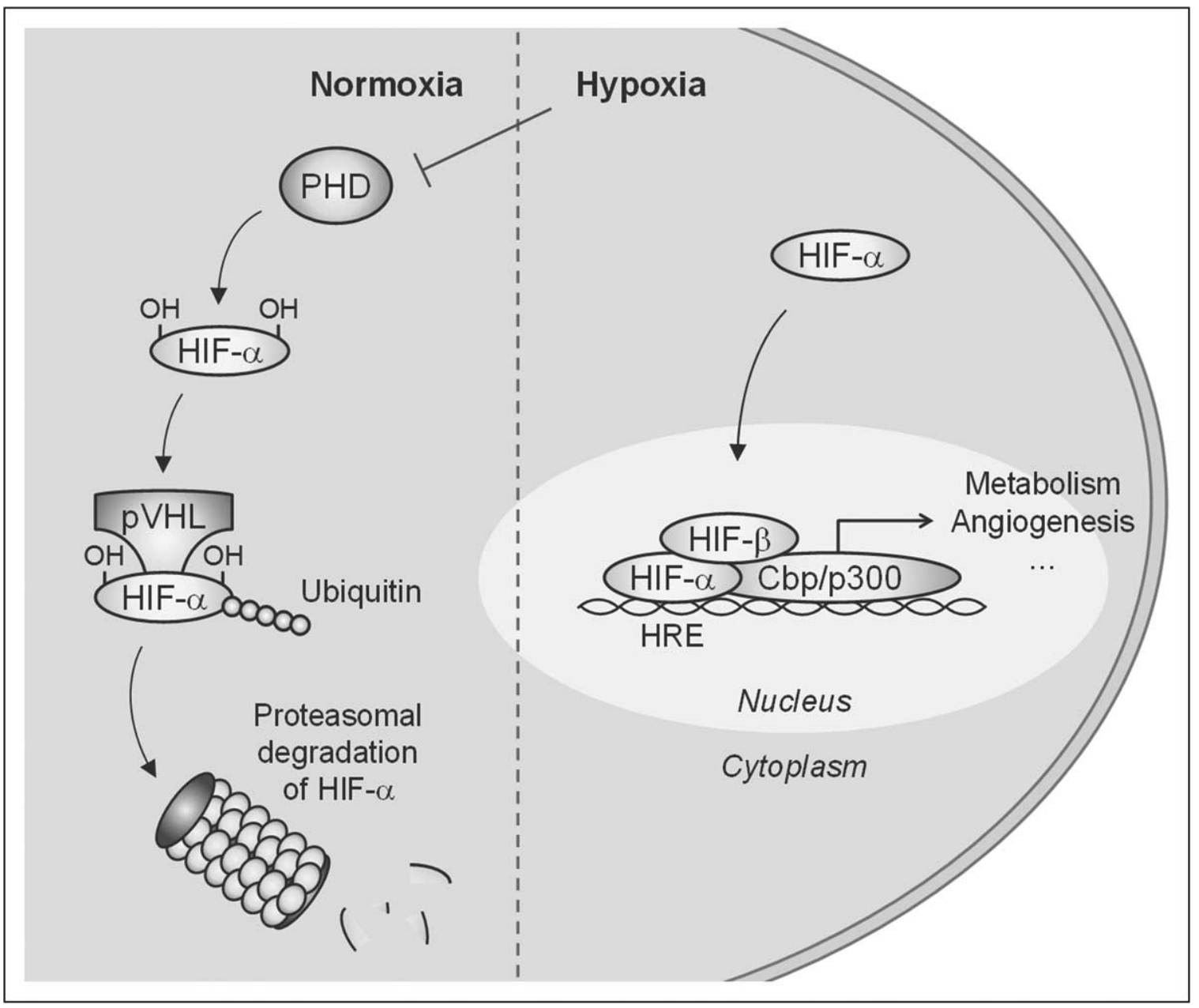

FIGURE 1. The hypoxia-inducible factor (HIF) signaling pathway. In normoxia, HIF prolyl hydroxylases (PHDs) hydroxylate specific proline residues of HIF- $\alpha$, leading to its proteosomal degradation mediated by the von Hippel-Lindau tumor-suppressor protein (pVHL). During hypoxia, PHDs become inactive and HIF- $\alpha$ translocates to the nucleus, where it forms a complex with $\mathrm{HIF}-\beta$ together with transcriptional coactivators $(\mathrm{Cbp} / \mathrm{p} 300)$ and binds to the hypoxia-responsive element (HRE) present in the promoter region of target genes. 
translocates to the nucleus, dimerizes with HIF- $\beta$ and, after recruitment of transcriptional co-activators, such as $\mathrm{Cbp} / \mathrm{p} 300$, binds to hypoxia response elements (HREs) within the promoter region of hypoxia-responsive genes (Fig. 1). Activation of HIF signaling influences cell and tissue function at multiple levels in physiological and pathological circumstances, but primarily intends to restore the decreased oxygen tension by stimulating angiogenesis, as well as enables cells to survive the hypoxic insult by adjusting cellular metabolism.

All skeletal cell types, including chondrocytes, osteoblasts, osteocytes and osteoclasts express the major components of the hypoxia-signaling pathway, and an accumulating body of evidence highlights its importance for regulating several steps during bone development, homeostasis and repair.

\section{HYPOXIA, HYPOXIA-INDUCIBLE TRANSCRIPTION FACTORS AND PROLYL HYDROXYLASES DURING BONE DEVELOPMENT}

Embryonic skeletal development starts with the condensation of mesenchymal stem cells (MCSs). Although the flat bones of the skull develop from mesenchymal cells that directly differentiate into bone-forming osteoblasts (i.e. intramembranous ossification), the other bones are derived from the replacement of a cartilaginous template by bone tissue $[1,2]$. This latter process is called endochondral ossification and several studies have demonstrated that the hypoxia signaling pathway is a critical regulator [10].

\section{Hypoxia-inducible transcription factor signaling controls early limb development}

The formation of long bones through endochondral ossification is initiated when pluripotent MSCs aggregate and form condensations prior to commitment to the chondrogenic lineage $[11,12]$. During these initial stages, the limb vasculature undergoes a remodeling process that renders the condensing mesenchyme avascular and thus hypoxic [13-15].

Deletion of Hif- $1 \alpha$ from paired related homeobox 1 (Prx1)-expressing cells in the limb bud mesenchyme did not affect mesenchymal condensation but delayed the chondrogenic differentiation from mesenchymal cells and the terminal differentiation of chondrocytes $[14,15]$. The underlying mechanism is not yet fully understood, but likely involves HIF-dependent regulation of SOX9, the key chondrogenic transcription factor in the developing autopod [14]. In contrast to HIF- $1 \alpha$, only a transient delay in skeletal development was observed in mice lacking HIF- $2 \alpha$ globally or in the limb bud $[16,17]$.
The role of the hypoxia signaling pathway during limb development was further investigated by conditional inactivation of $p V h l$, causing accumulation of both HIF- $1 \alpha$ and HIF- $2 \alpha$. Deletion of pVHL in Prx1-expressing limb bud cells did not affect MSCto-chondrocyte transition, but rather impaired chondrocyte proliferation, survival and terminal differentiation, resulting in shortening of the limbs [18]. The underlying molecular mechanisms, as well as the role of the PHD oxygen sensors in this process, are still unclear and require further investigation.

Taken together, the hypoxia signaling pathway tightly regulates the early events during limb development, including MSC survival, and chondrocyte proliferation and differentiation, mainly through HIF- $1 \alpha$.

\section{The interplay between hypoxia-inducible transcription factors and prolyl hydroxylases determines chondrocyte function in the developing growth plate}

After mesenchymal condensation, MSCs in the central region differentiate into chondrocytes, which start to proliferate and form the cartilaginous template for future bone deposition. Later, the centrally localized chondrocytes stop proliferating, become hypertrophic and, together with the surrounding osteoprogenitor cells, start producing proangiogenic growth factors that stimulate blood vessel ingrowth $[11,12]$. Together with the invading vessels, Osterix-positive progenitors move along into the cartilage template [19], which marks the onset of the development of the primary ossification center (POC). Whether hypoxic stress is involved in POC formation remains poorly studied. On the other hand, Runx 2 expressed by hypertrophic chondrocytes can stabilize HIF- $1 \alpha$ and drives blood vessel ingrowth in a hypoxia-independent manner [20].

Concomitant with the formation of the POC, chondrocytes become restrictedly localized at the opposing ends of the long bone. Within the developing growth plate, cell patterning towards the longitudinal axis occurs with proliferating chondrocytes localized at the bone ends and hypertrophic chondrocytes situated towards the diaphysis. As the fetal growth plate further expands in the absence of blood vessels, its center becomes increasingly hypoxic and HIF- $1 \alpha$ has been proposed as a crucial regulator of chondrocyte survival [21,22] (Fig. 2). Indeed, conditional deletion of Hif- $1 \alpha$ from type II collagen (COL2)-expressing chondrocytes resulted in severe cell death especially in the central region [21], and several mechanisms have been linked to the impaired survival. Because of the avascular nature of the growth plate, hypoxic chondrocytes activate HIF signaling to induce a proangiogenic 


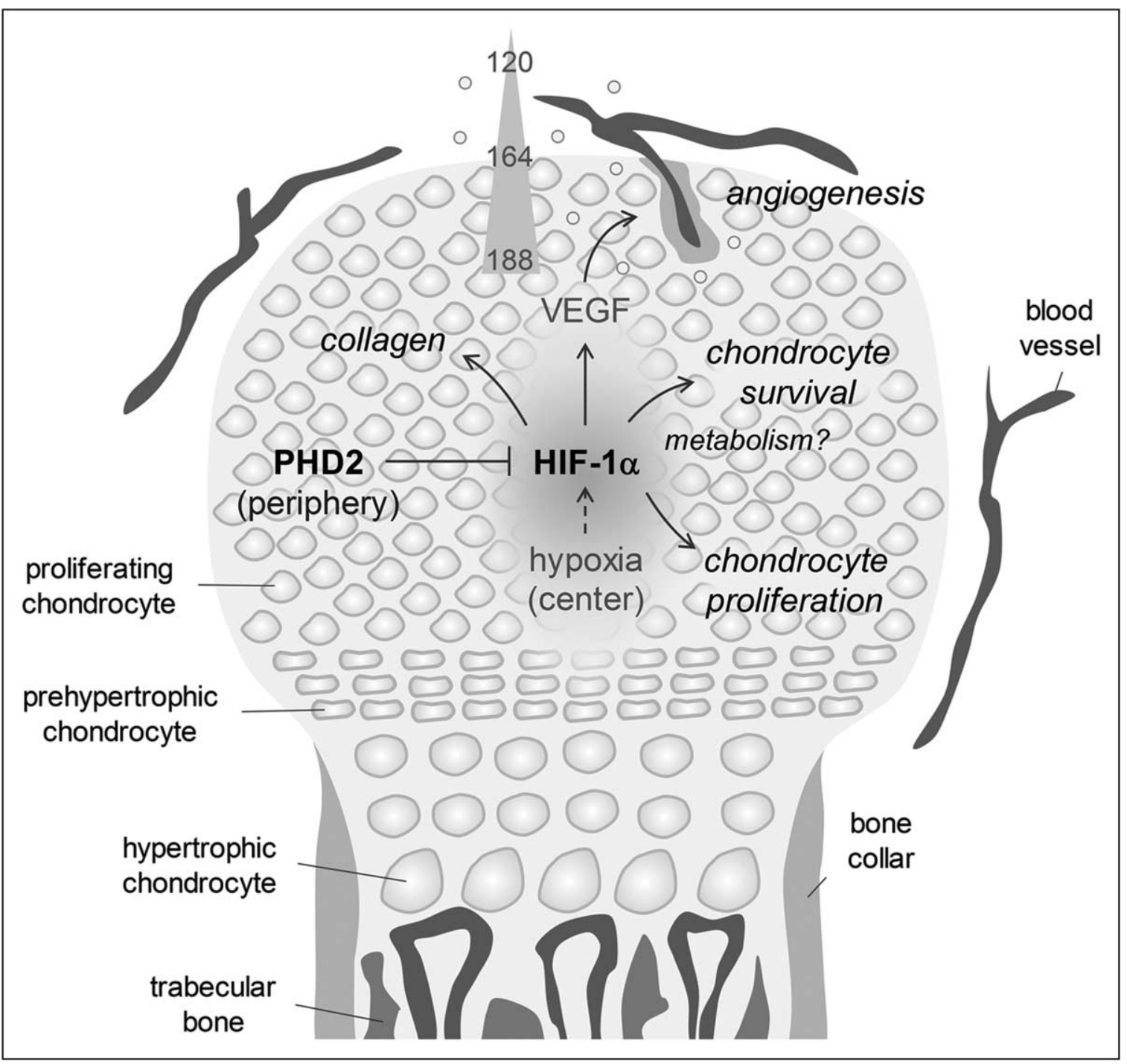

FIGURE 2. Hypoxia-inducible factor (HIF) signaling in the developing growth plate. HIF-1 $\alpha$ controls cell survival in centrally localized, hypoxic growth plate chondrocytes, by regulating vascular ingrowth and possibly also by metabolic adaptations. In addition, HIF signaling controls chondrocyte proliferation and ensures adequate collagen synthesis and modification. In the periphery of the growth plate, PHD2 activity is required to avoid undesirable HIF-1 $\alpha$ stabilization that would otherwise lead to metabolically induced skeletal dysplasia. VEGF, vascular endothelial growth factor.

response in order to improve oxygen and nutrient supply (Fig. 2). Vascular endothelial growth factor (VEGF), the most potent mitogen for endothelial cells and a direct HIF target gene [23], is expressed by proliferative and prehypertrophic chondrocytes [24-27]. Interestingly, a cell death phenotype was observed in mice with a conditional deletion of Vegf in chondrocytes or combined systemic deletion of VEGF120 and VEGF164 $[28,29]$. Chondrocyte cell death was, however, less pronounced than in Hif-1 $\alpha$ conditional knockout mice, suggesting that HIF regulates survival, at least in part, through VEGF signaling and the resulting vascular invasion. Indeed, chondrocyte cell death in Hif-1 $\alpha$ mutant mice could not be fully rescued by transgenic
VEGF164 expression [22], pointing at HIF-dependent cell-autonomous regulation of cell survival. HIF- $1 \alpha$ potentially adapts chondrocyte metabolism to survive the hypoxic insult (Fig. 2), as it regulates the expression of the glycolytic enzyme phosphoglycerate kinase 1 [22], but genetic proof is lacking. Moreover, HIF-1 $\alpha$ controls chondrocyte proliferation as well as matrix synthesis and modification [21,30-32]. However, the proliferation effect is observed mainly at the periphery of the growth plate, where HIF- $1 \alpha$ is only moderately expressed and may thus be a compensatory response induced by the cell death phenotype in the center.

The localized activation of the hypoxia signaling pathway in the center of the developing growth 
plate suggests that HIF levels have to be tightly regulated in the other avascular regions. Recently, we demonstrated that PHD2-regulated HIF-1 $\alpha$ inactivation is necessary to avoid metabolically induced skeletal dysplasia (Fig. 2). Indeed, inappropriate HIF$1 \alpha$ activation by PHD2 deletion decreases glucose oxidation, which limits chondrocyte proliferation and longitudinal bone growth. In addition, a glutamine-derived increase in $\alpha \mathrm{KG}$ results in over-modification of collagen and increased bone mass [33"-]. In line with our results, deletion of pVHL in COL2expressing chondrocytes reduces cell proliferation and increases matrix deposition in the developing growth plate [32], further supporting that regulatory mechanisms to keep correct HIF levels are critical for normal bone development.

Thus, HIF- $1 \alpha$ functions as a survival factor for centrally localized chondrocytes in the developing growth plate. Yet, despite the avascular environment, HIF levels have to be tightly controlled to avoid metabolically induced skeletal dysplasia.

\section{MAINTENANCE OF POSTNATAL BONE HOMEOSTASIS BY HYPOXIA-INDUCIBLE TRANSCRIPTION FACTORS AND PROLYL HYDROXYLASES}

Although adult bone has an inert appearance, it is constantly renewed by a process called remodeling, mediated by osteoclast-mediated bone resorption and osteoblast-controlled bone formation $[1,2]$. In contrast to the growth plate, the bone marrow is highly vascularized and these blood vessels supply bone cells with oxygen and nutrients to support their function, while also serving as a niche for hematopoietic and osteogenic stem/progenitor cells [3]. Despite the fact that bone is a highly vascularized organ, particular regions of the bone microenvironment are characterized by remarkably low oxygen tensions $[4,5]$.

The hypoxia signaling pathway in osteogenic cells has been implicated in the stimulation of angiogenesis and the coupling between osteogenic and angiogenic processes [10] (Fig. 3). Conditional deletion of Hif- $1 \alpha$ in osteoprogenitors or mature osteoblasts decreased bone volume and blood vessel number, the latter caused by a down-regulation of local VEGF production [34-37]. Conversely, activation of HIF signaling through pVHL or PHD deletion in osteolineage cells resulted in the opposite phenotype $\left[34,36,37,38^{*}, 39-41,42^{*}\right]$. The changes in vascular density have been proposed as an important driver but osteolineage cell-specific HIF signaling also controls bone homeostasis and bone cell function independent of an increase in angiogenesis as shown by recent studies. First, stabilization of
HIF-1 $\alpha$ in Osterix-positive osteoprogenitor cells in postnatal mice resulted, as expected, in high trabecular bone mass, which was associated by an increase in bone vascularization [39]. The high bone mass phenotype was, however, not secondary to increased angiogenesis, but was rather dependent on the up-regulation of glycolysis in osteoblast precursors as pharmacological block of this pathway, but not conditional deletion of Vegf, completely reversed the HIF- $1 \alpha$ dependent bone formation [39] (Fig. 3). How exactly the increased glycolysis contributes to osteoblast differentiation and bone formation remains still unknown. Second, activation of the hypoxia signaling pathway in osteogenic cells also affects the cross-talk with other skeletal cells, further fine-tuning HIF-dependent regulation of bone homeostasis (Fig. 3). Indeed, combined inactivation of PHD2 and PHD3 in Osterix-positive progenitors increased bone mass via an osteoprotegerin (OPG)-dependent, HIF-driven decrease in osteoclastogenesis [43]. Moreover, activation of HIF signaling in osteocytes epigenetically repressed sclerostin expression, resulting in a high-bone mass phenotype caused by increased bone formation and decreased bone resorption $\left[38^{*}, 44\right]$. Last, exacerbated HIF signaling in osteoprogenitors results in a high bone mass phenotype, together with an increase in hematopoietic stem cells (HSCs) and selective expansion of the erythroid lineage. Indeed, genetic ablation of $p V h l$ or combined deletion of $P h d 1 / 2 / 3$; or pharmacological inhibition of PHDs increased the number of $\mathrm{cKit}^{\text {high }} \mathrm{Lin}^{\text {low }} \mathrm{Sca} 1^{+}$ CD $150^{+} \mathrm{CD}^{2} 8^{-}$HSCs, which showed improved long-term repopulation in lethally irradiated mice. In addition, mutant mice developed polycythemia, which was caused by a HIF-dependent increase in osteoblastic erythropoietin (EPO) production [36]. Of note, the increase in HSCs in this model might be partially angiogenesis-dependent, as activation of HIF signaling in osteoprogenitors resulted in VEGF-mediated expansion of the bone marrow vasculature [36,39], a critical component of the HSC niche [45].

Recent data indicate that increased HIF signaling in osteogenic cells may directly, or indirectly through EPO induction, regulate fibroblast growth factor 23 (FGF23) levels [46] (Fig. 3). Normally, FGF23 is mainly produced by osteocytes and this hormone decreases in the kidney phosphate reabsorption and the production of $1,25(\mathrm{OH})_{2} \mathrm{D}$, the active form of vitamin $\mathrm{D}$. The latter effect is caused by decreased $1 \alpha$-hydroxylase expression and increased 24-hydroxylase levels. Factors involved in mineral homeostasis are considered to be the major regulators of FGF23 production, with phosphate, $1,25(\mathrm{OH})_{2} \mathrm{D}$ and parathyroid hormone being 


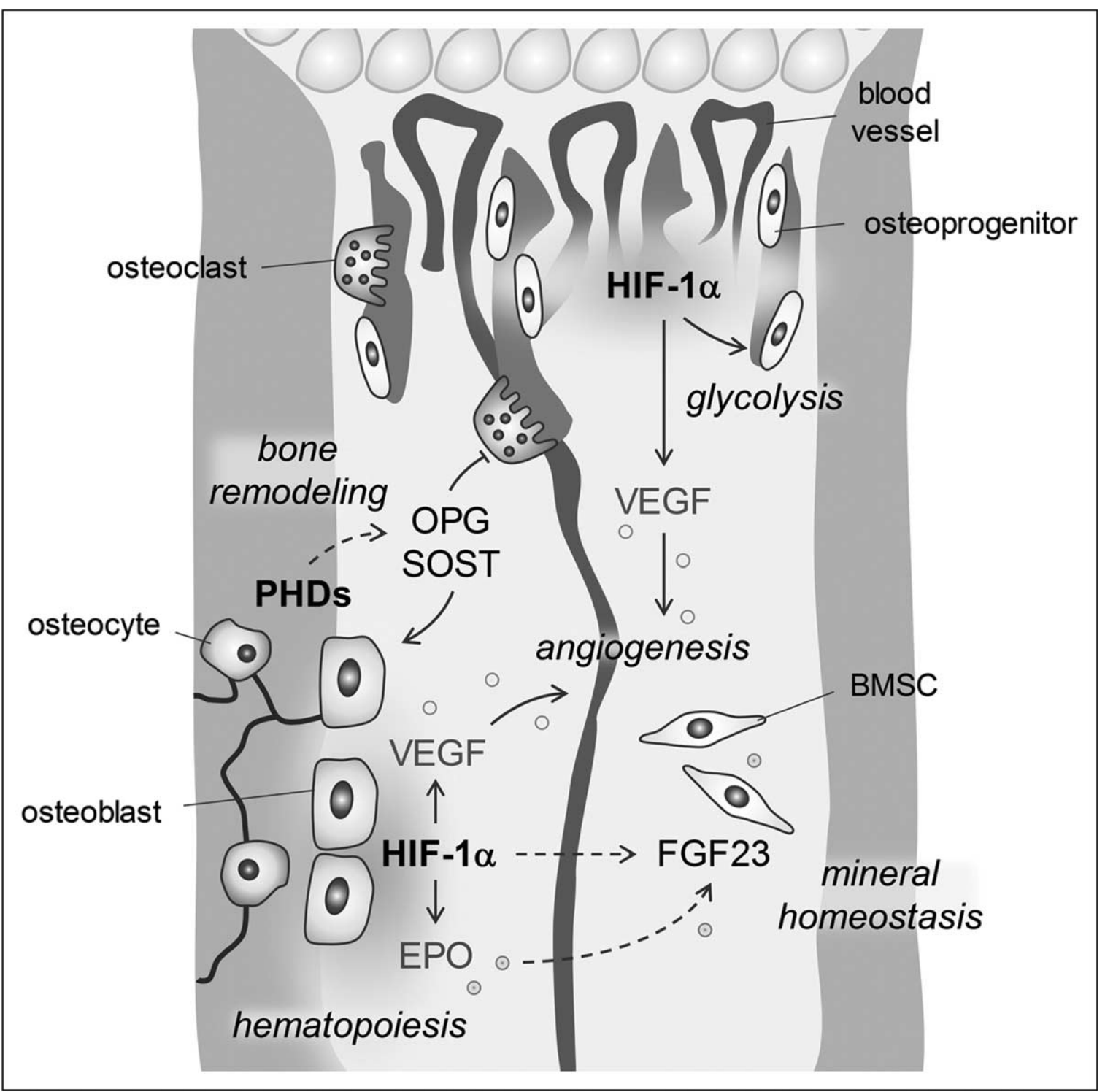

FIGURE 3. Hypoxia-inducible factor (HIF) signaling in postnatal bone homeostasis. HIF-dependent vascular endothelial growth factor (VEGF) production in osteogenic cells attracts blood vessels, which in turn stimulates bone formation by delivering oxygen, nutrients and possibly osteoprogenitors, as well as through the secretion of osteogenic factors. Moreover, osteoprogenitors require HIF-driven glycolysis to support bone formation and PHD activity in mature osteoblasts/osteocytes controls bone turnover by regulating osteoprotegerin (OPG) and sclerostin (SOST) secretion. In addition, erythropoietin (EPO) secreted by osteoblasts is an important regulator of bone hematopoiesis. Finally, EPO regulates FGF23 expression and cleavage in bone marrow cells (BMSC) and may thereby contribute to mineral and bone homeostasis, especially in situations of high EPO levels.

positive regulators [46]. However, nonmineral factors, including HIF and EPO signaling, are increasingly being recognized to regulate FGF23 production and its posttranslational cleavage. Only the intact FGF23 is bioactive, whereas the role of the cleaved fragments is not fully understood. HIF- $1 \alpha$, which may be induced by inflammation or iron deficiency [47], can bind to the Fgf23 promoter and increases its expression in osteogenic cells [48]. A disease with high bioactive FGF23 levels is $\mathrm{X}$-linked hypophosphatemia and this phenotype is mimicked in the Hyp mouse model. Inactivation of HIF-1 $\alpha$ signaling in mature osteoblasts of Hyp mice did neither alter serum intact FGF23 levels nor the bone phenotype, suggesting no link between osteoblastic HIF and FGF23 in this model [49]. However, the role of HIF signaling in other osteolineage cells as well as nonosseous cells could not be excluded. In addition to direct transcriptional regulation, HIF- $1 \alpha$ may also increase FGF23 levels by inducing EPO production (Fig. 3). EPO increases Fgf23 transcription and FGF23 cleavage, as the increases in 
circulating intact FGF23 levels are disproportionately less than the increases in circulating total FGF23 levels [50,51",52",53,54"]. In some studies, the increases in serum FGF23 levels are associated with reduced serum phosphate or $1,25(\mathrm{OH})_{2} \mathrm{D}$ levels, which in turn may alter bone homeostasis, although further confirmation is needed [51",52"]. EPO exerts this effect on $\mathrm{F} g \mathrm{f} 23$ expression by targeting nonosseous cells in the bone marrow, including cells from the erythroid lineage and hematopoietic progenitor cells $\left[51^{*}, 52^{*}, 53,54^{\prime \prime}\right]$. However, the mechanism by which EPO regulates FGF23 transcription and cleavage is not fully elucidated. A similar association between EPO and FGF23 levels is observed in humans, showing a larger increase in total FGF23 levels than in intact FGF23 levels $\left[51^{*}, 52^{*}, 53,4^{\prime \prime}\right]$. Whether this increase in FGF23 fragments is of pathophysiological significance has still to be elucidated. This novel interaction between EPO and FGF23 is especially important in patients with chronic kidney disease, which receive chronic EPO treatment.

Taken together, HIF signaling in osteolineage cells is required for normal bone function, as it directly regulates osteoblast function through metabolic adaptations and indirectly influences bone marrow endothelial cells, osteoclasts and hematopoietic cells by local secretion of VEGF, OPG, sclerostin and EPO, which may modify FGF23 levels and thereby mineral homeostasis.

\section{CONCLUSION}

Several studies have shown that HIF signaling is critical for normal bone development and homeostasis, especially to protect the skeletal cells against deleterious hypoxic effects [10]. Increased angiogenesis and adaptations in metabolism are contributing mechanisms, but comprehensive insight in the HIFdriven metabolic rewiring in osteoblasts is still lacking. In chondrocytes, extensive HIF signaling has to be avoided as the metabolic changes become maladaptive and induce skeletal dysplasia. In addition to regulating bone development and bone mass, several novel targets of HIF signaling in skeletal cells have recently been identified that affect other cell types, including osteoprotegerin that reduces osteoclastogenesis and EPO that stimulates erythropoiesis. In addition to its role in regulating hematopoietic lineages, EPO may increase the production and the cleavage of FGF23, although the mechanism remains to be determined. In addition, the contribution of osteogenic HIF signaling in this EPO-FGF23 pathway requires further in-vivo validation using mouse models with osteoblast and/or renal-specific inactivation of HIF or PHD. These insights will be important for the clinical translation of PHD inhibitors as treatment for anemia-related chronic kidney disease.

\section{Acknowledgements}

None.

\section{Financial support and sponsorship}

G.C. acknowledges funding from the Research Foundation-Flanders (FWO: G.0964.14, G.0A42.16 and GOB34.18). S.S. is a postdoctoral fellow from the FWO (12H5917N).

\section{Conflicts of interest}

There are no conflicts of interest.

\section{REFERENCES AND RECOMMENDED READING}

Papers of particular interest, published within the annual period of review, have been highlighted as:

- of special interest

m. of outstanding interest

1. Harada S, Rodan GA. Control of osteoblast function and regulation of bone mass. Nature 2003; 423:349-355.

2. Long F. Building strong bones: molecular regulation of the osteoblast lineage. Nat Rev Mol Cell Biol 2011; 13:27-38.

3. Stegen $\mathrm{S}$, Carmeliet $\mathrm{G}$. The skeletal vascular system - breathing life into bone tissue. Bone 2018; 115:50-58.

4. Spencer JA, Ferraro F, Roussakis E, et al. Direct measurement of local oxygen concentration in the bone marrow of live animals. Nature 2014; 508:269-273

5. Chow DC, Wenning LA, Miller WM, Papoutsakis ET. Modeling $\mathrm{pO}(2)$ distributions in the bone marrow hematopoietic compartment. II. Modified Kroghian models. Biophys J 2001; 81:685-696.

6. Ramasamy SK, Kusumbe AP, Schiller M, et al. Blood flow controls bone vascular function and osteogenesis. Nat Commun 2016; 7:13601.

7. Ivan M, Kaelin WG Jr. The EGLN-HIF O2-sensing system: multiple inputs and feedbacks. Mol Cell 2017; 66:772-779.

8. Pugh CW, Ratcliffe PJ. New horizons in hypoxia signaling pathways. Exp Cell Res 2017; 356:116-121.

9. Prabhakar NR, Semenza GL. Oxygen sensing and homeostasis. Physiology (Bethesda) 2015; 30:340-348.

10. Maes C, Carmeliet G, Schipani E. Hypoxia-driven pathways in bone development, regeneration and disease. Nat Rev Rheumatol 2012; 8:358-366.

11. Karsenty $G$, Wagner EF. Reaching a genetic and molecular understanding of skeletal development. Dev Cell 2002; 2:389-406.

12. Kronenberg HM. Developmental regulation of the growth plate. Nature 2003; 423:332-336.

13. Hallmann $\mathrm{R}$, Feinberg $\mathrm{RN}$, Latker $\mathrm{CH}$, et al. Regression of blood vessels precedes cartilage differentiation during chick limb development. Differentiation 1987; 34:98-105.

14. Amarilio R, Viukov SV, Sharir A, et al. HIF1alpha regulation of Sox 9 is necessary to maintain differentiation of hypoxic prechondrogenic cells during early skeletogenesis. Development 2007; 134:3917-3928.

15. Provot $S$, Zinyk D, Gunes $Y$, et al. Hif-1alpha regulates differentiation of limb bud mesenchyme and joint development. J Cell Biol 2007; 177:451-464.

16. Saito T, Fukai $A$, Mabuchi $A$, et al. Transcriptional regulation of endochondral ossification by HIF-2alpha during skeletal growth and osteoarthritis development. Nat Med 2010; 16:678-686.

17. Araldi $E$, Khatri R, Giaccia AJ, et al. Lack of HIF-2alpha in limb bud mesenchyme causes a modest and transient delay of endochondral bone development. Nat Med 2011; 17:25-26.

18. Mangiavini $L$, Merceron $C$, Araldi $E$, et al. Loss of $V H L$ in mesenchymal progenitors of the limb bud alters multiple steps of endochondral bone development. Dev Biol 2014; 393:124-136.

19. Maes $C$, Kobayashi T, Selig MK, et al. Osteoblast precursors, but not mature osteoblasts, move into developing and fractured bones along with invading blood vessels. Dev Cell 2010; 19:329-344.

20. Lee $\mathrm{SH}$, Che $\mathrm{X}$, Jeong $\mathrm{JH}$, et al. Runx2 protein stabilizes hypoxia-inducible factor-1 alpha through competition with von Hippel-Lindau protein ( $\mathrm{pVHL}$ ) and stimulates angiogenesis in growth plate hypertrophic chondrocytes. J Biol Chem 2012; 287:14760-14771. 
21. Schipani E, Ryan HE, Didrickson S, et al. Hypoxia in cartilage: HIF-1alpha is essential for chondrocyte growth arrest and survival. Genes Dev 2001; 15:2865-2876

22. Maes $\mathrm{C}$, Araldi $\mathrm{E}$, Haigh $\mathrm{K}$, et al. VEGF-independent cell-autonomous functions of HIF-1alpha regulating oxygen consumption in fetal cartilage are critical for chondrocyte survival. J Bone Miner Res 2012; 27:596-609.

23. Pugh CW, Ratcliffe PJ. Regulation of angiogenesis by hypoxia: role of the HIF system. Nat Med 2003; 9:677-684.

24. Gerber $\mathrm{HP}, \mathrm{Vu} T \mathrm{TH}$, Ryan $\mathrm{AM}$, et al. VEGF couples hypertrophic cartilage remodeling, ossification and angiogenesis during endochondral bone formation. Nat Med 1999; 5:623-628.

25. Zelzer E, Glotzer DJ, Hartmann C, et al. Tissue specific regulation of VEGF expression during bone development requires Cbfa1/Runx2. Mech Dev 2001; 106:97-106.

26. Maes $C$, Carmeliet $P$, Moermans $K$, et al. Impaired angiogenesis and endochondral bone formation in mice lacking the vascular endothelial growth factor isoforms VEGF164 and VEGF188. Mech Dev 2002; 111:61-73

27. Zelzer E, McLean W, Ng YS, et al. Skeletal defects in VEGF(120/120) mice reveal multiple roles for VEGF in skeletogenesis. Development 2002; 129:1893-1904.

28. Zelzer E, Mamluk R, Ferrara N, et al. VEGFA is necessary for chondrocyte survival during bone development. Development 2004; 131:2161-2171.

29. Maes $C$, Stockmans I, Moermans $K$, et al. Soluble VEGF isoforms are essential for establishing epiphyseal vascularization and regulating chondrocyte development and survival. J Clin Invest 2004; 113:188-199.

30. Bentovim L, Amarilio R, Zelzer E. HIF1 alpha is a central regulator of collagen hydroxylation and secretion under hypoxia during bone development. Development 2012; 139:4473-4483.

31. Aro $\mathrm{E}$, Khatri R, Gerard-O'Riley R, et al. Hypoxia-inducible factor-1 (HIF-1) but not HIF-2 is essential for hypoxic induction of collagen prolyl 4-hydroxylases in primary newborn mouse epiphyseal growth plate chondrocytes. J Biol Chem 2012; 287:37134-37144.

32. Pfander D, Kobayashi T, Knight MC, et al. Deletion of Vhlh in chondrocytes reduces cell proliferation and increases matrix deposition during growth plate development. Development 2004; 131:2497-2508.

33. Stegen $\mathrm{S}$, Laperre $\mathrm{K}$, Eelen $\mathrm{G}$, et al. HIF-1 alpha metabolically controls collagen

- synthesis and modification in chondrocytes. Nature 2019; 565:511-515.

This study reports that the PHD2 oxygen sensor is required to avoid undesirable

HIF signaling in growth plate chondrocytes, that would otherwise lead to meta-

bolically induced skeletal dysplasia.

34. Wang $Y$, Wan $C$, Deng $L$, et al. The hypoxia-inducible factor alpha pathway couples angiogenesis to osteogenesis during skeletal development. J Clin Invest 2007; 117:1616-1626.

35. Shomento SH, Wan C, Cao X, et al. Hypoxia-inducible factors 1 alpha and 2alpha exert both distinct and overlapping functions in long bone development. J Cell Biochem 2010; 109:196-204

36. Rankin EB, Wu C, Khatri R, et al. The HIF signaling pathway in osteoblasts directly modulates erythropoiesis through the production of EPO. Cell 2012; 149:63-74.

37. Devignes CS, Aslan $Y$, Brenot $A$, et al. HIF signaling in osteoblast-lineage cells promotes systemic breast cancer growth and metastasis in mice. Proc Natl Acad Sci U S A 2018; 115:E992-E1001.

38. Stegen S, Stockmans I, Moermans K, et al. Osteocytic oxygen sensing

- controls bone mass through epigenetic regulation of sclerostin. Nat Commun 2018; 9:2557.

This study describes the role of PHD2 in mediating the cross-talk between osteocytes, osteoblasts and osteoclasts through epigenetic regulation of sclerostin expression. The authors demonstrate that the HIF-driven increase in bone mass was independent of changes in angiogenesis, but was rather caused by a SIRT1-dependent decrease in sclerostin.
39. Regan JN, Lim J, Shi Y, et al. Up-regulation of glycolytic metabolism is required for HIF1alpha-driven bone formation. Proc Natl Acad Sci U S A 2014; 111:8673-8678.

40. Stegen S, van Gastel N, Eelen G, et al. HIF-1alpha promotes glutaminemediated redox homeostasis and glycogen-dependent bioenergetics to support postimplantation bone cell survival. Cell Metab 2016; 23: 265-279.

41. Rauner M, Franke $K$, Murray $M$, et al. Increased EPO levels are associated with bone loss in mice lacking PHD2 in EPO-producing cells. J Bone Miner Res 2016; 31:1877-1887.

42. Dirckx N, Tower RJ, Mercken EM, et al. Vhl deletion in osteoblasts boosts

- cellular glycolysis and improves global glucose metabolism. J Clin Invest 2018; 128:1087-1105.

This study shows that active HIF signaling in osteoprogenitors promotes glycolysis and thereby regulates blood glucose levels.

43. Wu $\mathrm{C}$, Rankin $\mathrm{EB}$, Castellini $\mathrm{L}$, et al. Oxygen-sensing PHDs regulate bone homeostasis through the modulation of osteoprotegerin. Genes Dev 2015; 29:817-831.

44. Loots GG, Robling AG, Chang JC, et al. Vhl deficiency in osteocytes produces high bone mass and hematopoietic defects. Bone 2018; 116: 307-314.

45. Morrison SJ, Scadden DT. The bone marrow niche for haematopoietic stem cells. Nature 2014; 505:327-334.

46. Hanudel MR, Laster M, Salusky IB. Nonrenal-related mechanisms of FGF23 pathophysiology. Curr Osteoporos Rep 2018; 16:724-729.

47. David V, Martin A, Isakova $T$, et al. Inflammation and functional iron deficiency regulate fibroblast growth factor 23 production. Kidney Int 2016; 89:135-146.

48. Zhang $\mathrm{Q}$, Doucet $\mathrm{M}$, Tomlinson $\mathrm{RE}$, et al. The hypoxia-inducible factor-1alpha activates ectopic production of fibroblast growth factor 23 in tumor-induced osteomalacia. Bone Res 2016; 4:16011.

49. Hum JM, Clinkenbeard EL, Ip C, et al. The metabolic bone disease associated with the Hyp mutation is independent of osteoblastic HIF1alpha expression. Bone Rep 2017; 6:38-43.

50. Flamme I, Ellinghaus $P$, Urrego $D$, Kruger T. FGF23 expression in rodents is directly induced via erythropoietin after inhibition of hypoxia inducible factor proline hydroxylase. PLoS One 2017; 12:e0186979.

51. Clinkenbeard EL, Hanudel MR, Stayrook KR, et al. Erythropoietin stimulates

- murine and human fibroblast growth factor-23, revealing novel roles for bone and bone marrow. Haematologica 2017; 102:e427-e430.

This study demonstrates that EPO administration in mice and anemic patients increases serum FGF23 levels.

52. Daryadel $A$, Bettoni $C$, Haider $T$, et al. Erythropoietin stimulates fibroblast

growth factor 23 (FGF23) in mice and men. Pflugers Arch 2018; 470: $1569-1582$

This study shows that acute administration of recombinant EPO in healthy humans and mice increases the C-terminal fragment of FGF23, but not or only with a delay intact FGF23. In mice, this effect was associated with decreased $1,25(\mathrm{OH})_{2}$ vitamin D levels.

53. Rabadi S, Udo I, Leaf DE, et al. Acute blood loss stimulates fibroblast growth factor 23 production. Am J Physiol Renal Physiol 2018; 314: F132-F139.

54. Hanudel MR, Eisenga MF, Rappaport M, et al. Effects of erythropoietin on

- fibroblast growth factor 23 in mice and humans. Nephrol Dial Transplant 2018. [Epub ahead of print]

This study reports that mice with high endogenous EPO levels have disproportionaly high levels of total FGF23 compared to intact FGF23, suggesting increased FGF23 production and proteolytic cleavage. Moreover, the authors provide clinical evidence that in CKD patients, serum EPO levels are positively and independently associated with total FGF23 levels. 